\title{
Production of mixture more resistance from some vegetable oils for frying process
}

\begin{abstract}
Olive, sunflower oils and binary oil mixtures were continuously heated at $180^{\circ} \mathrm{C}$ for $16 \mathrm{hr}$. Aliquots of potato chips were fried in the aforementioned oil samples. Effect of deep-fat frying on these blends was studied by assessing the changes in percent fat absorption and physico-chemical properties during repeated frying process (four times). The physico-chemical properties like refractive index, color, smoke point, acid value, peroxide value, polar content and oxidative stability by Rancimat method and fatty acid composition of olive, sunflower oils and binary oil mixtures were evaluated. In addition, organoleptic tests were performed on fried chips. Organoleptic results for fried potato chips indicate that all types of chips obtained from heated oils were categorized good. In general, the results suggest that olive oil alone and in mixtures with sunflower oil have to ban its use in frying process.
\end{abstract}

Keywords: olive oil, oxidative stability, frying process, fatty acids
Volume I Issue 4 - 2015

\author{
Amany M Basuny,' Shaker M Arafat, ${ }^{2}$ Hoda A \\ Farag,' Hanaa M Soliman ${ }^{3}$ \\ 'Food Science \& Nutrition Department, King Faisal University, \\ Saudi Arabia \\ ${ }^{2}$ Oils \& Fats Research Department, Food Technology Research \\ Institute, Egypt \\ ${ }^{3}$ Fats and Oils Department, National Research Centre, Egypt
}

Correspondence: Amany M Basuny, Food Science \& Nutrition Department, King Faisal University, Saudi Arabia, Tel 00201060732315, Email dramany_basuny@yahoo.com

Received: November 02, 2015 | Published: December 21 , 2015

\section{Introduction}

Frying is one of the most popular culinary process worldwide, both for industrial and domestic food preparation procedures. Fried products have unique organoleptic and sensorial proprieties, including flavor, texture and appearance, which turn them largely enjoyed by consumers. Also, this procedure considerably reduces cooking time ${ }^{1}$ and is regarded as including equal or even smaller nutrient losses when compared with other common culinary process. ${ }^{2}$

Fat contents in fried products become a great concern among consumers as taking an excess fat may contribute to overweight and obesity. As much 300 million people in the world population, as well as $64 \%$ of the United States adult population are considered either overweight or obese ${ }^{3}$ and this percentage has increased over the last four decades. It has been concluded that, a large number of medical dysfunctions have been associated with obesity/overweight including osteoarthritis, diabetes, cancer, cardiovascular and liver diseases. ${ }^{4}$ Nowadays, many fast food outlets are using vegetable frying medium to produce fried products, such as fried chicken, burgers and potato chips. All these products absorb oils between 6-15\%. Potato chips will absorb oil up to $14 \%$ when frying in palm olein. ${ }^{5}$ A similar pattern on oil up take is also observed when using different frying medium, including soybean oil, canola oil and sunflower oil. Many techniques and treatments are used in order to reduce oil up take in fried products and improve the frying performance of the oil.

Once of the common techniques is by using blending. ${ }^{6}$ Olive oil is typically the main lipid source in the Mediterranean diet, being used and salad dressing and for frying purposes. Its beneficial properties are associated with the richness in monounsaturated fatty acids, but other minor compounds also take an important part. ${ }^{7,8}$ The differences to other common vegetable oils are enhanced by the fact that olive oil is mostly obtained to refining-virgin olive oil-retaining therefore higher amount of important bioactive components of the olive fruit. More recently, however, mostly supported by economic reasons and reduced consumer information, the consumption of olive oil a blend of refined with virgin olive oils in undeclared properties, is increasing, together with other refined vegetable oils blends.

Several studies support a relationship between the Mediterranean diet and s lower incidence of some important diseases of our century, including cancer ${ }^{9}$ and Cardiovascular disease. ${ }^{10,11}$

The high temperature used during frying, in the presence of oxygen and water, induce important chemical changes of the oils, namely by oxidation, polymerization, crystallization, and hydrolysis, ${ }^{12,13}$ inevitably reducing their shelf life and affecting directly the quality of the final fried food. ${ }^{14}$ These chemical reactions are influenced by the type and quality of the oil. The food properties, and the food/oil ratio, among other parameters, ${ }^{13}$ altogether determining the frying oil performance. Each vegetable oil is characterized by typical stabilities against oxidation, dependent on the fatty acids compositions, particularly the unsaturation degree, and the content and composition of minor compounds such as to copherol, certain sterol, hydrocarbons, carotenoids and polyphenols. ${ }^{14}$

The present study was designed to investigate the effect of deepfat frying on physico-chemical properties of virgin olive oil blends, besides that, assessment of the sensory qualities and oil uptake of fried potato chips also be carried out.

\section{Materials and methods}

\section{Oils}

i. Refined sunflower oil was obtained from Savola Edible Oil Company (Al-Dammam, Saudi Arabia). The oil peroxide and acid values were 0.60 (meq.active oxygen $/ \mathrm{kg}$ oil) and $0.07(\mathrm{mg} \mathrm{KOH} / \mathrm{g}$ oil), respectively.

ii. Extra virgin olive oil was obtained from Al-Jouf Company (Al-Jouf Saudi Arabia). The peroxide and acid values of oil were 2.10(meq. active oxygen $/ \mathrm{kg}$ oil) and $0.10(\mathrm{mg} \mathrm{KOH} / \mathrm{g}$ oil), respectively. 


\section{Solvents and reagents}

All solvents and reagents were of analytical, chromatographic or spectroscopic and were supplied from Merck (Darmstadt, Germany). All experiments and analytical determination were performed at least in duplicate.

\section{Frying process}

A known amount $(2 \mathrm{~L})$ of each refined sunflower and olive oil were separately placed in a carbon Bleu deep fat-frying (Model DZo5D, China) equipped with a temperature controller-portions of olive oil (system 1) were mixed with sunflower oil (system 2) at ratios of 60:40(system 3), 40:60 (system 4) and 20:80 (system 5),(V/V). Potato (2mm thicknessX40mm widthX50mm length) were soaked in a sodium chloride solution $(10 \%, \mathrm{w} / \mathrm{v})$ for $30 \mathrm{~min}$ and fried in different oil samples at $180^{\circ} \mathrm{C} \pm 5^{\circ} \mathrm{C}$. The frying process was conducted $4 \mathrm{hr}$ each day and the frying period was 4 consecutive days. Oil samples were taken every $4 \mathrm{hr}$ and the total continuous heating period was $16 \mathrm{hr}$. The oil samples were left to cool down ten stored at $10^{\circ} \mathrm{C}$ for analysis. The fried potato chips samples were collected each day at the end of frying process and were kept at ambient temperature for sensory evaluation and oil uptake analysis.

\section{Sensory evaluation}

For sensory evaluation, sufficient samples of freshly fried potato chips were taken on the day 1 , and 4 of frying experiment and then served to trained panelists within $10 \mathrm{~min}$ after taken out of the fryer. Panelists within $10 \mathrm{~min}$ after taken out of the fryer. Panelists were requested to evaluate the organoleptic attributes, i.e. color, odor, oiliness, crispiness, taste and overall acceptance) of fried potato chips. A nine-point hedonic scoring scale was used by the panel members to judge their perception, where $9=$ extremely good and 1 extremely bad. The sensory scores will be analyzed statistically using SPSS software (version 18.0 for windows, SPSS onc., Chicago, IL, USA).

\section{Physical and chemical parameters evaluated}

Refractive index, acid value and peroxide value were determined according to. ${ }^{15}$ Smoke point refers to the temperature at which the oil sample begins to smoke and is recorded as outlined by Nielson. ${ }^{16}$ A Lovi bond Tintometer apparatus (The tintometer Ltd., Salisbury, England) was applied to measure the color of fresh and fried oil samples. The yellow slides were fixed at 35 and the intensity of red glass was assigned through matching with the oil samples. ${ }^{16}$ The total polar content of oil samples were determined according to the economical micro method developed by Schulte. ${ }^{17}$

\section{Oil uptake analysis}

Total oil content of fried potato chips was determined by Soxhlet extraction according to AOAC. ${ }^{15}$ The oil was extracted using a Soxtec ${ }^{\text {tm }}$ 2050 Auto Fat Extraction System (Foss Analytical AB, Hoganas, Sweden). Two gram fried potato chips sample was extraction for $2 \mathrm{~h}$ using n-hexane as a solvent.

\section{Total phenol content}

Total phenols were isolated from a solution of oil in n-hexane by triple-extraction with water-methanol $(60: 40 \mathrm{v} / \mathrm{v})$, and estimated with Folin-Ciocalteu reagent at $735 \mathrm{~nm}$. Results were expressed as $\mathrm{mg}$ of caffeic acid per $\mathrm{kg}$ of oil. ${ }^{8}$

\section{Oxidative stability (Rancimat)}

The oxidative stability was estimated by measuring the oxidation induction time, on a Rancimat apparatus (Metrohm CH series 679). Air ( $20 \mathrm{~L} / \mathrm{h}$ was bubbled through the oil $(5.0 \mathrm{~g})$ heated at $100^{\circ} \mathrm{C} \pm 2^{\circ} \mathrm{C}$, with the volatile compounds being collected in water, and the increasing water conductivity continually measured. The time taken to reach the conductivity inflection was recorded. ${ }^{18}$

\section{Fatty acids composition of fried sunflower, olive oils and their binary mixtures}

Capillary gas chromatograph (HP 6890) was used for the qualitative and quantitative determinations of fatty acids of the oil samples and reported in relative area percentages. Fatty acids were transesterfied into their corresponding fatty acid methyl esters by shaking a solution of oil $(0.1 \mathrm{~g})$ in heptane $(2 \mathrm{ml})$ with solution methanolic potassium hydroxide $(0.2 \mathrm{ml}, 2 \mathrm{~N})$. The fatty acid methyl esters were identified using a gas chromatograph equipped with DB-23(5\%-cyanopropylmethyl polysiloxane) capillary column $(60 \mathrm{mx} 0.32 \mathrm{mmX} 0.25 \mu \mathrm{m}$ film thickness) and flame ionization detector. Nitrogen flow rate was $0.6 \mathrm{ml} / \mathrm{min}$, hydrogen and air-flow rates were 45 and $450 \mathrm{ml} / \mathrm{min}$, respectively. The oven temperature was isothermally heated $195^{\circ} \mathrm{C}$. The injector and the detector temperatures were $230^{\circ} \mathrm{C}$ and $250^{\circ} \mathrm{C}$, respectively. Fatty acid methyl esters were identified by comparing their retention times with known fatty acid standard mixture. Peak areas were automatically computed by an integrator. All GC measurements for each oil sample were made in triplicate and the averages were reported.

\section{Statistical analysis}

All experiments and measurements were carried out in triplicate, and the data were suggested to analysis of variance (ANOVA). Analysis of variance and regression analyses were performed according to the M Stat Cand Excel software. Significant differences between means were determined by Duncan's multiple range tests. P values less than 0.05 were considered statistically significant.

\section{Results and discussion}

\section{Changes in refractive index}

Figure 1 shows the refractive index values for non-fried and fried olive oil, sunflower oil and their blends (60:40, 40:60 and 80:20 v/v). The values demonstrate that olive oil refractive index value was lower than that of the refractive index of sunflower oil and blend oil samples. There is a strong relationship between the refractive index and iodine value, with higher iodine values would have higher refractive index and this fact is in line with data of the present work. The value of refractive index of fried olive oil, sunflower oil and blends of them indicate a linear relationship between their refractive indices and frying period. The increase of the refractive index values over frying time for the oil systems was in the order: sunflower oil $>$ olive oil + sunflower oil $(40: 60)>$ olive oil + sunflower oil $(60: 40)$ olive oil+sunflower oil $(80: 20)>$ olive oil. This sequence is in line index values for the conjugated compounds are higher than that of their non-conjugated isomers. It is established that during oil frying some of the non-conjugated double bonds are converted to conjugated ones and this process cause an increase in the refractive index value. ${ }^{19}$ 


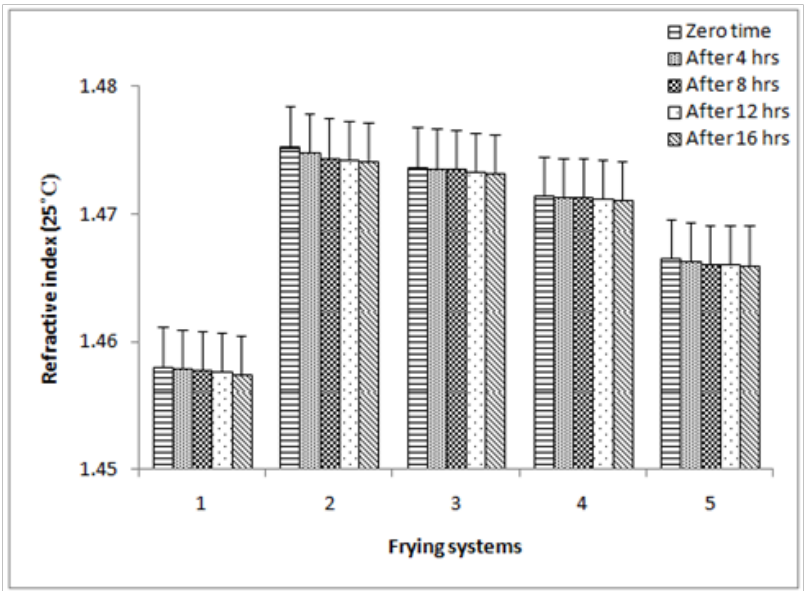

Figure I Refractive index of blended olive oil during frying. Values are expressed as mean $\pm S E, P \geq 0.05$.

\section{Changes in color}

In most cases, two types of colored glasses of Lovebird Tintometer, i. e., yellow and red, were used to measure the color of the oils. The yellow glasses were fixed at a value of 35 and the variation in oil color was matched with red glasses. Figure 2 illustrate that the initial red colors for olive oil and sunflower oil were 2.20 and 1.70 , respectively. As a general trend, the intensity of the red color in all oil systems was increased as the frying time increased. Accordingly blending sunflower oil with olive produced lighter frying media.

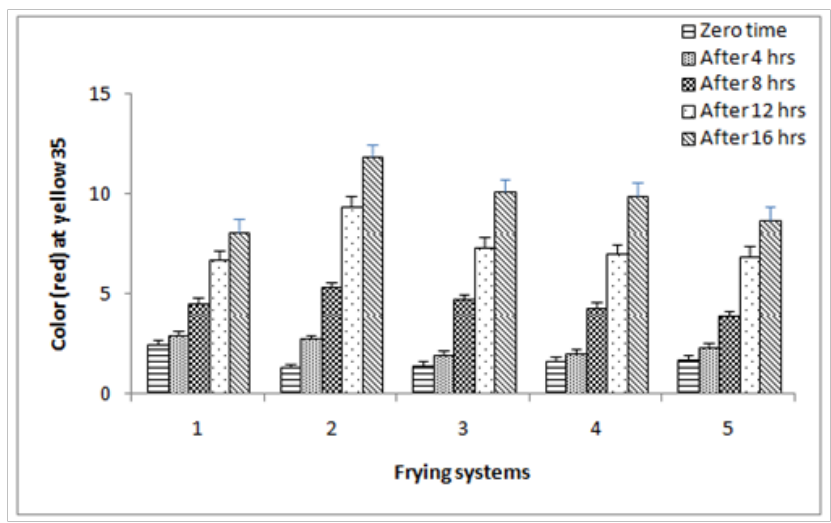

Figure 2 Color of blended olive oil during frying. Values are expressed as mean $\pm S E, P \geq 0.05$

\section{Changes in smoke point}

Figure 3 shows the changes in smoke point of fried oils and their blends at various periods compared with those at zero time. Value of smoke point of fried olive oil was gradually decrease compared with sunflower oil. It is worth noting that the smoke points of fried olive oil mixed sunflower oil at variance levels were generally higher than olive oil alone.

\section{Changes in the free fatty acids}

The free fatty acids, resulting mainly from hydrolysis if triglycerides, was evaluated by conventional acidity measurement. Virgin olive oil presented acidity level below $0.3 \%$ before frying, in accordance with the labeling category-extra virgin olive oils $(\leq 0.8 \%)$. Sunflower oil acidity was comparatively smaller $(0.1 \%)$, due to the neutralization step during refining, and entirely within the legal limits for vegetable oils, as defined in Codex Standard 210(2009). The acid value of fried oils showed gradually increase with frying time. Hence, the increase of the acid value was in the order: sunflower oil $>$ sunflower oil + olive oil $(40: 60)>$ sunflower oil + olive oil $(20: 80)$ sunflower oil + olive oil $(60: 40)>$ olive oil (Figure 4$)$. The improvement in sunflower oil quality heating at $180^{\circ} \mathrm{C} \pm 5^{\circ} \mathrm{C}$ when mixed with olive oil. Despite the inexistence of mandatory rejection points for used oil regarding hydrolysis, ${ }^{12}$ this parameters is some timed used in rapid commercial tests as an estimation of the rejected point. Based on the information obtained from these frying experiments, the hydrolysis was reduced at the rejection point, being a parameter with minor capacity to evaluate the true thermal degradation of frying oils.

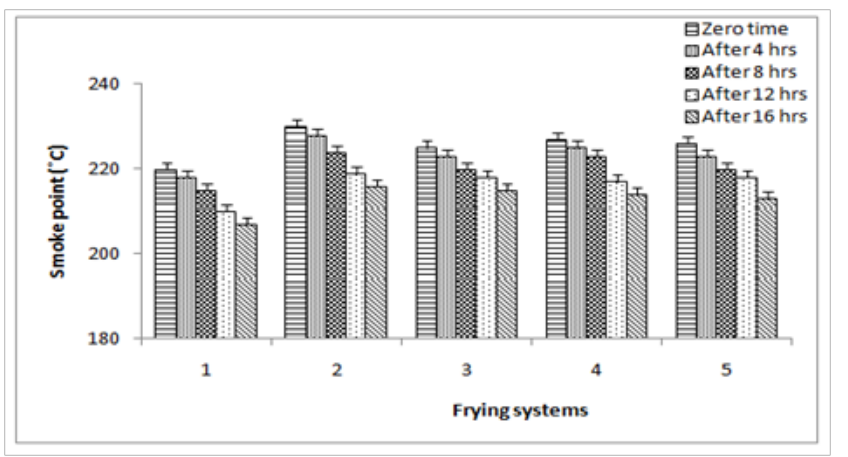

Figure 3 Smoke point of blended oil during frying. Values are expressed as mean $\pm S E, P \geq 0.05$.

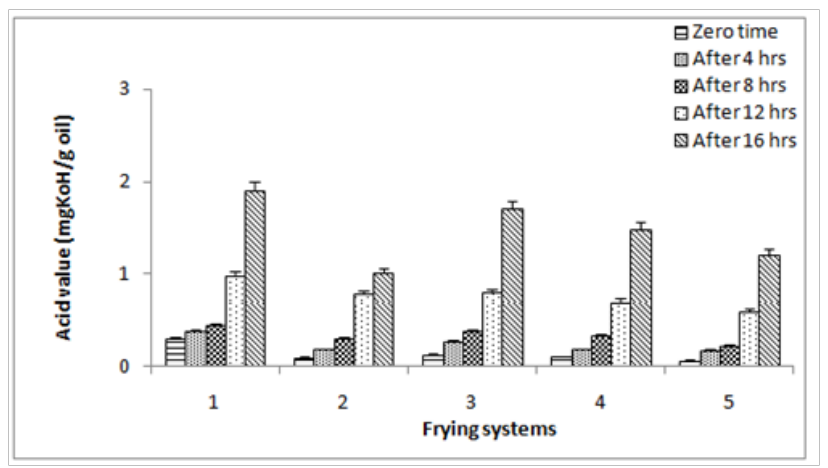

Figure 4 Acid value of blended olive oil during frying. Values are expressed as mean $\pm S E, P \geq 0.05$.

\section{Changes in peroxide value}

The hydro peroxides formation is correlated with the fatty acids oxidation susceptibility and the antioxidant levels, however, hydro peroxides are transient chemical compounds and, despite being a mandatory parameter for both olive and sunflower oils oxidative status, they are not always directly correlated with sample oxidation, mostly on heated samples. The results for peroxide value levels are detailed in Figure 5. All unheated samples presented peroxide values with the legal limits: below 20 meq. $\mathrm{O}_{2} / \mathrm{kg}$ for virgin olive oil, $10 \mathrm{meq}$. $\mathrm{O}_{2} / \mathrm{kg}$ for sunflower oil and 10meq. $\mathrm{O}_{2} /$ for the blends of sunflower oil with virgin olive oil at different levels. The peroxide values of these oils were within the recommended values for human consumption. 
The changes in the peroxide values of fried olive oil, sunflower oil and their blends are shown in. Figure 5 he values of peroxide value of the fried oils were progressively and significantly increased during frying process. The values of peroxide value for the oils at the end of frying period indicate that the increase of peroxide value was in the order: olive oil $>$ olive oil + sunflower oil $(80: 20)>$ olive oil + sunflower oil $(60: 40)>$ olive oil+sunflower oil $(40: 60)>$ sunflower oil. The higher peroxide levels in the sunflower oil, being related with the formation of oxidation products, can also give some insight into the higher total polar content observed in this sample. The similarity in the olive oil samples behaviors and its opposition to the sunflower oil blend indicates that the fatty acid composition might be the main responsible.

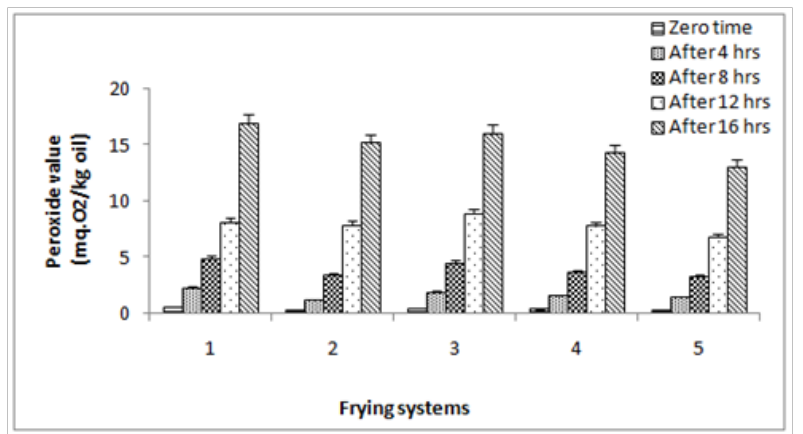

Figure 5 Peroxide value of blended olive oil during frying.Values are expressed as mean $\pm S E, P \geq 0.05$.

\section{Changes in the total polar compounds}

In accordance with the Portuguese law and as mandatory in several countries, the legal rejection point for frying oils is $25 \%$ of total polar content, including under this label the hydrolysis products (diglycerides, monoglycerides and free fatty acids), the oxidation and polymeric derivatives, all formed at temperature below $180^{\circ} \mathrm{C}$ (Portaria No. 1135/95). Under real frying facilities, mostly restaurants and industries, this parameter is usually evaluated by commercial rapid testes, mostly based on colorimetric reading, which have proven to correlate well with the values obtained by official standard, and allow rapid on-line measurements. Changes in polar content of olive oil, sunflower oil and mixtures of them are shown in Figure 6. At zero time, non- detectable polar compounds were found. Frying of olive oil, sunflower oil and their mixtures at $180^{\circ} \mathrm{C} \pm 5^{\circ} \mathrm{C}$ for $4 \mathrm{hr} / 4$ day caused increase in polar compounds content of all oil systems. The increases of polar compound contents of oil systems were in sunflower oil $>$ sunflower oil+olive oil $(60: 40)>$ sunflower+olive oil $(40: 60)>$ sunflower oil+olive oil $(20: 80)>$ olive oil. In addition blending olive oil with sunflower oil induced lowering effect on the formation of total polar content.

\section{Changes in oxidative stability}

The Rancimat method is frequently used for evaluating or predicting oxidative stabilities under heating conditions, known as induction time. Despite being an important tool for comparison of different vegetable oils, the results are not directly transposable to real frying conditions. Clearly distinct results were observed for all samples (Figure 7). The highest oxidative stability was depicted by the olive oil while sunflower oil the lowest oxidative stability. The effect of frying time on the oils under study shows a gradual and significant decrease in the oxidative stability of the oils.

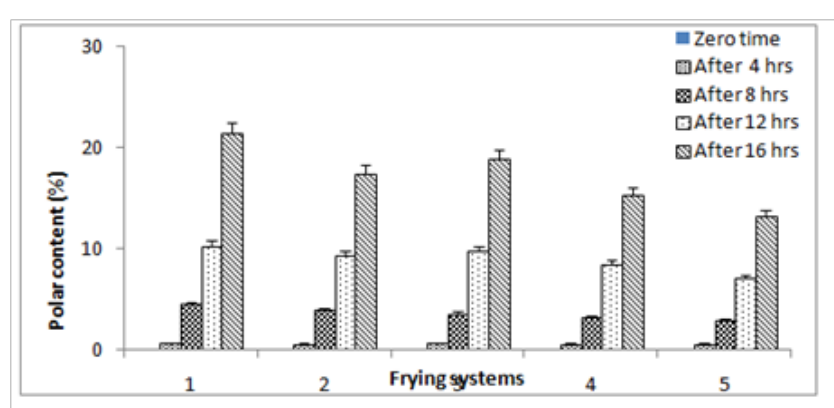

Figure 6 Polar contents of blended olive oil during frying.Values are expressed as mean $\pm S E, P \geq 0.05$.

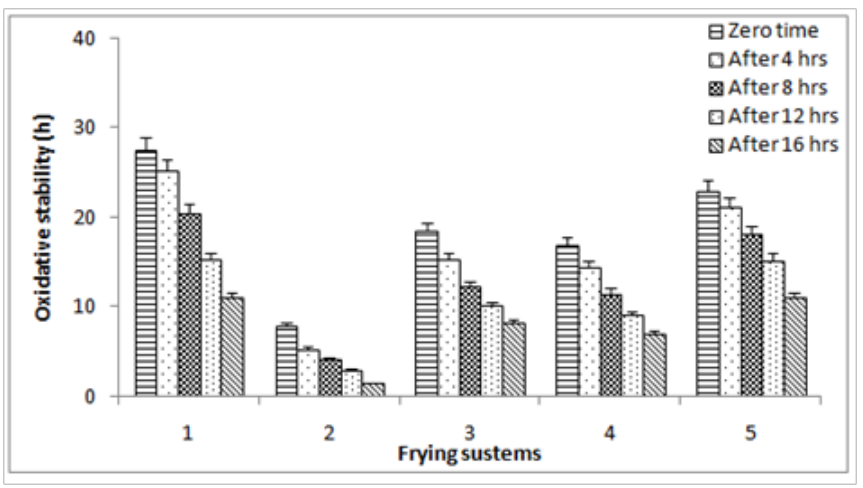

Figure 7 Oxidative stability of blended olive oil during frying. Values are expressed as mean $\pm S E, P \geq 0.05$.

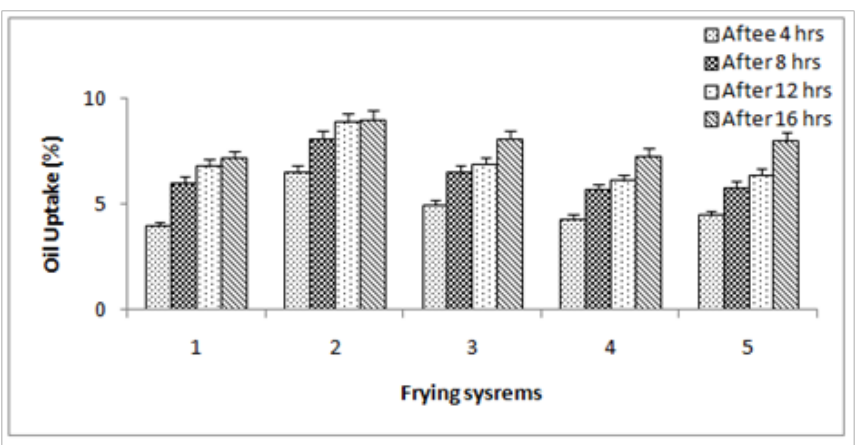

Figure 8 Oil uptake of fried potato chips during frying experiment using blended olive oil.Values are expressed as mean $\pm S E, P \geq 0.05$.

\section{Total phenol content}

In comparison with other vegetable oils and olive oil are receiving particular attention for their phenolic content given that these compounds have antioxidant and antimicrobial effects. ${ }^{20}$ Indeed, phenols are frequently described as key factors in olive oils oxidative resistance. ${ }^{21}$ The amount of total phenols, expressed as $\mathrm{mg}$ of caffeic acid equivalents, was higher in the olive oil, with $255.00 \mathrm{mg} / \mathrm{kg}$, while sunflower oil was lower $69.00 \mathrm{mg} / \mathrm{kg}$, respectively. Therefore, if these compounds take a part in the frying resistance towards oxidation. The olive oil fatty acid composition, highly monounsaturated, should be the rate limiting factor determining its oxidation. The almost absence of protective phenols and the polyunsaturated nature are certainly important factors contributing to the precocious oxidation of the blend (sunflower oil). 


\section{Changes in the fatty acid composition}

In order to understand the chemical issues behind the frying susceptibility observed for the samples under analysis, several parameters were evaluated. Being the fatty acids the main oils constituent and their nature, particularly the unsaturation degree, among the most important factors determining the oxidative stability, the fatty acid composition was evaluated in detail. Olive oil, as expected, was characterized by a high degree of monounsaturated fatty acids, particularly oleic acid, an important issue contributing to the cardiovascular health effects. ${ }^{10}$ The sunflower oil was characterized by a dominant polyunsaturated faction, particularly linolenic acid, and a comparatively low amount of oleic acid and linoleic acid. When comparing the relative percentage of fatty acids during the frying sessions, a clear reduction of unsaturated fatty acids was observed, with the consequent increase in the saturated fatty acids amount (Table 1). The decrease was particularly noticeable in the polyunsaturated fatty acids, presumably by oxidation, with the linolenic acid reduced to about half the initial amounts, despite being a minor fatty acids. The sunflower oil alternative followed the same trend with equivalent losses for the same sampling times, despite the increased polyunsaturated amounts. A linear trans fatty acids increase with frying time observed for all samples. Based on these observations and despite the clear differences on the fatty acid composition of both matrices, the degradation rated appears to be similar.

Table I Change in the fatty acids composition during frying process. Values are expressed as mean $\pm S E, P \geq 0.05$

\begin{tabular}{|c|c|c|c|c|c|}
\hline Frying Time (h) & SFA & C18:1 & C18:2 & C18:3 & TFA \\
\hline \multicolumn{6}{|l|}{ System 1} \\
\hline 0 & $14.50 \pm 0.80$ & $76.80 \pm 4.55$ & $5.64 \pm 0.20$ & $0.76 \pm 0.01$ & $0.03 \pm 0.001$ \\
\hline 1 & $14.39 \pm 0.74$ & $76.83 \pm 4.00$ & $5.60 \pm 0.19$ & $0.71 \pm 0.01$ & $0.06 \pm 0.001$ \\
\hline 2 & $14.95 \pm 0.82$ & $76.85 \pm 4.08$ & $5.13 \pm 0.22$ & $0.62 \pm 0.01$ & $0.14 \pm 0.001$ \\
\hline 3 & $14.80 \pm 0.79$ & $77.40 \pm 4.55$ & $5.04 \pm 0.18$ & $0.53 \pm 0.01$ & $0.17 \pm 0.001$ \\
\hline 4 & $14.43 \pm 0.81$ & $77.96 \pm 5.01$ & $4.86 \pm 0.15$ & $0.49 \pm 0.01$ & $0.15 \pm 0.001$ \\
\hline \multicolumn{6}{|l|}{ System 2} \\
\hline 0 & $11.28 \pm 0.64$ & $24.82 \pm 2.00$ & $62.78 \pm 3.33$ & $0.11 \pm 0.001$ & $0.27 \pm 0.01$ \\
\hline 1 & $11.38 \pm 0.66$ & $24.63 \pm 1.90$ & $62.70 \pm 3.45$ & $0.10 \pm 0.001$ & $0.29 \pm 0.01$ \\
\hline 2 & $11.35 \pm 0.63$ & $24.49 \pm 1.88$ & $62.81 \pm 3.13$ & $0.10 \pm 0.001$ & $0.31 \pm 0.01$ \\
\hline 3 & $11.43 \pm 0.61$ & $24.72 \pm 2.01$ & $62.43 \pm 3.01$ & $0.10 \pm 0.001$ & $0.34 \pm 0.01$ \\
\hline 4 & $11.66 \pm 0.60$ & $25.21 \pm 2.18$ & $61.56 \pm 3.19$ & $0.09 \pm 0.001$ & $0.38 \pm 0.01$ \\
\hline \multicolumn{6}{|l|}{ System 3} \\
\hline 0 & $13.50 \pm 0.71$ & $56.30 \pm 2.98$ & $50.10 \pm 2.50$ & $0.51 \pm 0.01$ & $0.04 \pm 0.01$ \\
\hline 1 & $13.60 \pm 0.74$ & $55.50 \pm 2.87$ & $49.33 \pm 2.64$ & $0.50 \pm 0.01$ & $0.09 \pm 0.01$ \\
\hline 2 & $13.20 \pm 0.70$ & $55.60 \pm 2.90$ & $48.50 \pm 2.37$ & $0.44 \pm 0.01$ & $0.10 \pm 0.01$ \\
\hline 3 & $13.13 \pm 0.71$ & $56.30 \pm 3.01$ & $47.80 \pm 2.56$ & $0.33 \pm 0.01$ & $0.15 \pm 0.01$ \\
\hline 4 & $13.81 \pm 0.78$ & $56.90 \pm 3.05$ & $47.30 \pm 2.23$ & $0.30 \pm 0.01$ & $0.21 \pm 0.01$ \\
\hline \multicolumn{6}{|l|}{ System 4} \\
\hline 0 & $12.40 \pm 0.65$ & $51.00 \pm 1.98$ & $49.00 \pm 2.00$ & $0.48 \pm .0 .03$ & $0.10 \pm 0.01$ \\
\hline 1 & $12.33 \pm 0.64$ & $51.50 \pm 2.09$ & $48.20 \pm 1.99$ & $0.45 \pm 0.02$ & $0.13 \pm 0.01$ \\
\hline 2 & $12.20 \pm 0.66$ & $51.30 \pm 2.33$ & $47.80 \pm 1.78$ & $0.40 \pm 0.01$ & $0.15 \pm 0.01$ \\
\hline 3 & $12.50 \pm 0.63$ & $52.30 \pm 2.74$ & $46.20 \pm 1.91$ & $0.38 \pm 0.01$ & $0.19 \pm 0.01$ \\
\hline 4 & $12.61 \pm 0.67$ & $53.00 \pm 2.81$ & $45.10 \pm 1.86$ & $0.35 \pm 0.01$ & $0.23 \pm 0.01$ \\
\hline \multicolumn{6}{|l|}{ System 5} \\
\hline 0 & $13.90 \pm 0.72$ & $70.00 \pm 4.88$ & $7.30 \pm 0.81$ & $0.60 \pm 0.03$ & $0.04 \pm 0.001$ \\
\hline 1 & $13.85 \pm 0.69$ & $70.50 \pm 4.66$ & $7.10 \pm 0.82$ & $0.52 \pm 0.02$ & $0.08 \pm 0.001$ \\
\hline 2 & $13.95 \pm 0.53$ & $70.90 \pm 4.19$ & $6.20 \pm 0.67$ & $0.41 \pm 0.02$ & $0.15 \pm 0.001$ \\
\hline 3 & $13.98 \pm 0.55$ & $71.00 \pm 4.90$ & $5.90 \pm 0.63$ & $0.38 \pm 0.01$ & $0.18 \pm 0.001$ \\
\hline 4 & $14.00 \pm 0.78$ & $71.30 \pm 4.82$ & $4.33 \pm 0.53$ & $0.31 \pm 0.01$ & $0.21 \pm 0.001$ \\
\hline
\end{tabular}




\section{Oil uptake analysis}

Figure 8 shows that the percentage of oil uptake/absorption by the fried potato chips $\mathrm{n}$ frying oils. By subtracting the initial oil content $(0.00 \%)$ in the raw potato chips, it was found that the oil uptake was the lowest in the potato fried chips using olive oil: sunflower oil $(60: 40)$ blend. As reported by Bastida et al. ${ }^{22}$ the fat absorption in fried products will be low when the amount of unsaturated fatty acids, i, e. linoleic acid in frying medium is low. Also, polymers cause higher oil absorption of foods and the oil rich in linoleic acid is more easily polymerized during deep-fat frying than the oil rich in oleic acid. ${ }^{23}$

\section{Sensory evaluation}

From the sensory mean scores on the day1, there were significant differences $(\mathrm{P} \geq 0.05)$ in terms of color, odor, taste, appearance, texture and overall quality in all samples (Figure 9). In day 4, the samples indicated that there was no significant difference in color, odor, appearance, while there was a significant differences $(\mathrm{P} \geq 0.05)$ in texture, taste and overall quality in all samples (Figure 10). Throughout this study, the addition of olive had affected the consumer perception on the fried potato chips. The sensory data generally showed that fried potato chips in olive oil were of significantly different $(\mathrm{P} \geq 0.05)$ from fried potato chips in sunflower oil.

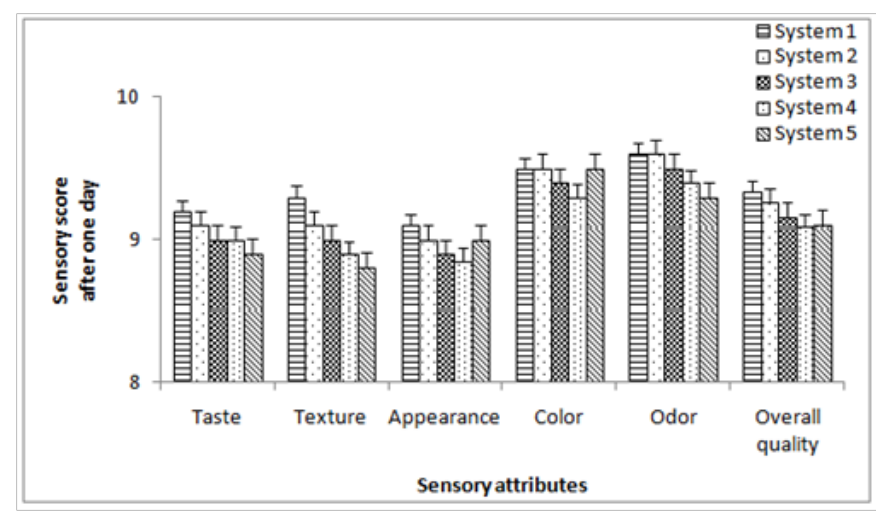

Figure 9 Mean sensory score of fried potato chips in blended olive oil of I day. Values are expressed as mean $\pm S E, P \geq 0.05$.

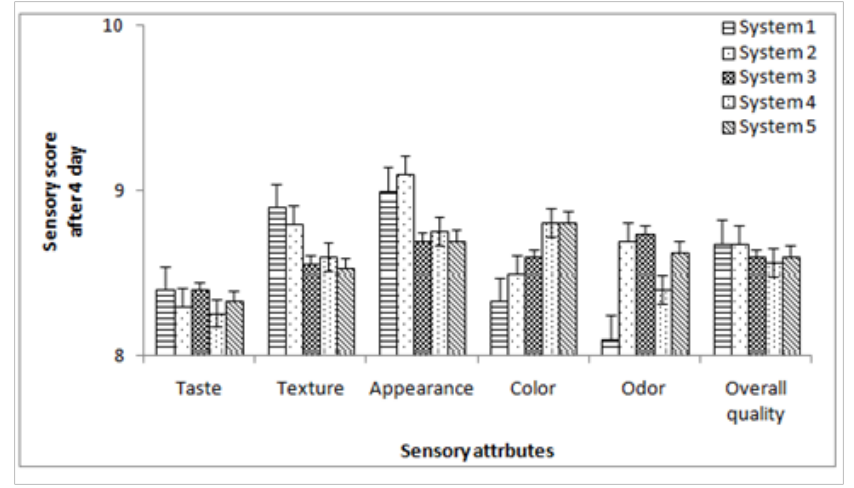

Figure I0 Mean sensory score of fried potato chips in blended olive oil of 4 day. Values are expressed as mean $\pm S E, P \geq 0.05$.

\section{Conclusion}

In the present work is proved that virgin olive oil can improve the frying performance of sunflower oil during deep-frying. The physic-chemical properties of olive oil with sunflower oil blends were better than sunflower oil alone. In term of oil uptake, the potato chips fried in olive oil with sunflower oil blends absorbed less oil than in sunflower oil. Moreover, sensory evaluation the addition of olive oil to sunflower oil showed significant differences on the oiliness, taste and overall acceptability of fried potato chips. Phenolic compounds seem to be key determining the reduced loss of oxidative stability and the highly monounsaturated nature of the olive oil is a second decisive parameter for the reduced thermal degradation under frying conditions. In overall, it can be concluded that the addition of olive oil to sunflower oil gave a major improvement in frying stability of oil.

\section{Acknowledgements}

None.

\section{Conflict of interest}

The author declares no conflict of interest.

\section{References}

1. Sanche muniz FJ, Bastida S. Effect of frying and thermal oxidation on olive oil and food quality. In: Quiles JL, et al. editors. Olive Oil and Human Health. Oxford Shire, UK: CAB International Publishing; 2006. p. 74-108.

2. Fillion L, Henry CJ. Nutrient losses and gains during frying: a review. Int J Food Sci Nutr. 1998;49(2):157-168.

3. Flegal KM, Carroll MD, Johnson CL. Prevalence and trends in obesity among US adults, 1999-2000. JAMA. 2002;288(14):1723-1727.

4. Bray G. Medical consequences of obesity. AJ Clin Endocrinol Metab. 2004;89(6):2583-2589.

5. Razali I, Badri M. Palm Oil Dev. 2003;38:12-15.

6. Omar M, Nor Hazwani M, Nazreen M, et al. Studies on frying quality of virgin coconut oil and shortening blends. Oriental Journal of Chemistry. 2014;30:1279-1286.

7. Varela G, Rui-Roso B. Some nutritional aspects of olive oil. In: Harwood J, et al. editors. Handbook of Olive Oil Analysis and Properties. Maryland, USA: Aspen Publisher Inc; 2000. p. 565-580.

8. Casal S, Malheiro R, Sendas A, et al. Olive oil stability under deep-frying conditions. Food Chem Toxicol. 2010;48(10):2972-2979.

9. Assmann G, De Backer G, Bagnara S, et al. International consensus statement on olive oil and the Mediterranean diet: implication for health in Europe. The olive oil and the mediterranean diet panel. Eur J Cancer Prev. 1997;6(5):418-421.

10. Covas MI. Olive oil and the cardiovascular system. Pharmacol Res. 2007;55(3):175-186.

11. Goulas V, Orphanides A, Pelava E, et al. Impact of thermal processing methods on polyphenols and antioxidant activity of olive oil polar fraction. Journal of Food Processing and Preservation. 2015;39(6):1919-1924.

12. Paul S, Mittal GC. Regulating the use of degraded oil/fat in deep-fat/oil food frying. Crit Rev Food Sci Nutr. 1997;37(7):636-662.

13. Saguy IS, Dana D. Integrated approach to deep-fat frying: engineering, nutrition, health and consumers aspects. Journal Food Engineering. 2003;56(3):143-152.

14. Kochhar SP. The composition of frying oils. In: Rossel JB editor. Frying Improving Quality. Cambridge, USA: Woodhead Publishing Ltd; 2001. p. 87-114.

15. OA C. Official Methods of Association of Agricultural Chemicals. 18th ed. Washington DC, USA: AOAC; 2005. 
16. Nielson SS. Food analysis. 2nd ed. Maryland, USA: Aspen publishers Inc; 1998. p. 222-223.

17. Schulte E. Economical micro method for determination of polar components in frying fats. European Journal of Lipid Science and Technology. 2004;106(11):772-776.

18. Farhoosh R. The effect of operational parameters of the Rancimat method on the determination of the oxidation stability measures and shelf-life prediction of soybean oil. Journal of the American Oil Chemists Society. 2007;84(3):205-209.

19. Baily's. Industrial oil and fat products. In: Hui YU editor. 5th ed. John Wiley and Sons, USA: Wiley internet Science Publication; 2006 pp. 499.

20. Ocakoglu D, Tokatli F, Ozen B, et al. Distribution of simple phenols, phenolic acids and flavonoids in Turkish monovarietal extra virgin olive oils for two harvest years. Food Chemistry. 2009;113(2):401-410.
21. Gómez Alonso S, Fregapane G, Salvador MD, et al. Changes in phenolic composition and antioxidant activity of virgin olive oil during frying. $J$ Agric Food Chem. 2003;51(3):667-672.

22. Bastida S, Sanche Muniz FJ. Thermal oxidation of olive oil, Sunflower oil and a mix of both oils during forty discontinuous domestic frying of different foods. Food Science Technology. 2001;7(1):15-21.

23. Tompkins C, Perkins EG. Frying performance of low linolenic acid soybean oil. Journal of American Oil Chemists Society. 2000;77(3):223-229. 